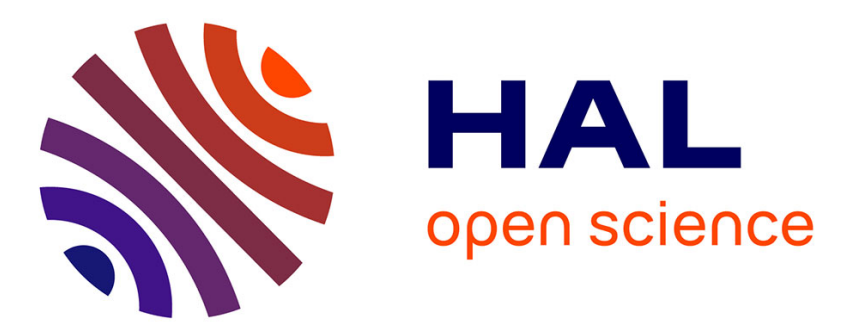

\title{
La classicisation des écrivaines à la Bibliothèque nationale de France : étude des politiques d'acquisition et de valorisation des œuvres produites par des femmes
}

\author{
Lilas Bass
}

\section{- To cite this version:}

Lilas Bass. La classicisation des écrivaines à la Bibliothèque nationale de France : étude des politiques d'acquisition et de valorisation des œuvres produites par des femmes. Culture et Musées, 2021, 38, pp.99-122. 10.4000/culturemusees.6802 . hal-03471489

\author{
HAL Id: hal-03471489 \\ https://hal.science/hal-03471489
}

Submitted on 8 Dec 2021

HAL is a multi-disciplinary open access archive for the deposit and dissemination of scientific research documents, whether they are published or not. The documents may come from teaching and research institutions in France or abroad, or from public or private research centers.
L'archive ouverte pluridisciplinaire HAL, est destinée au dépôt et à la diffusion de documents scientifiques de niveau recherche, publiés ou non, émanant des établissements d'enseignement et de recherche français ou étrangers, des laboratoires publics ou privés. 


\section{OpenEdition}

Journals

\section{Culture \& Musées}

Muséologie et recherches sur la culture

$38 \mid 2021$

Patrimonialisations de la littérature

Dossier

\section{La classicisation des écrivaines à la Bibliothèque nationale de France : étude des politiques d'acquisition et de valorisation des œuvres produites par des femmes}

Female authors become classics at the Bibliothèque Nationale de France: a study in acquisition policy and the promotion of women's works

La clasicización de las escritoras en la Biblioteca Nacional de Francia: análisis de las políticas de adquisición y puesta en valor de las obras producidas por mujeres

\section{LILAS BASS}

p. $99-122$

https://doi.org/10.4000/culturemusees.6802

\section{Résumés}

Français English Español

Cet article rend compte du rôle des bibliothécaires de la Bibliothèque nationale de France (BnF) dans la classicisation des écrivaines. Plus précisément, il revient sur les trajectoires des bibliothécaires étudiées, qui déterminent en partie leur ethos professionnel, entre "neutralité » et promotion d'une littérature légitime. Ces pratiques professionnelles propres à la $\mathrm{BnF}$ se confrontent néanmoins à certains espaces de socialisation, notamment militants, que les bibliothécaires convoquent dans leurs politiques d'acquisition et de valorisation par la voie d'une double justification : suivre la demande du lectorat et les demandes universitaires concernant les œuvres féminines - à plus forte raison dans un contexte post-\#MeToo. Ces politiques d'acquisition et de valorisation impliquent deux voies de classicisation des écrivaines : par la promotion de la littérature contemporaine, par la voie de la redécouverte d'auteures oubliées, à l'instar des pratiques militantes de la deuxième vague féministe en France. 
This article exposes the role played by librarians at the Bibliothèque nationale de France (BnF) in rendering female authors classics. Specifically, it proposes to examine the trajectory of the librarians being studied in that it partly determines their professional ethos, caught between "neutrality" and the promotion of legitimate literature. These professional practices, specific to the BnF, are nonetheless confronted with certain social spaces, particularly that of activism, to which librarians may refer in their acquisitions policy as well as in the promotion of certain works by means of a twofold justification: to stay up to date with readers' demands as well as those from academic spheres regarding literature by female authors, all the more so in a post-\#MeToo era. These acquisition and promotional policies are carried out through two processes destined to make female-authored literature classic: namely the promotion of contemporary literature, and the rediscovery of forgotten female authors in the manner of activists in French second-wave feminism.

Este artículo expone el papel de las bibliotecarias de la Biblioteca Nacional de Francia (BnF) en la clasicización de las escritoras. Específicamente, examina las trayectorias de las bibliotecarias estudiadas, quienes sitúan una parte de su ethos profesional, entre la "neutralidad" y la promoción de la literatura legítima. Estas prácticas profesionales propias de la BnF se confrontan, sin embargo, con ciertos espacios de socialización, particularmente militantes, que las bibliotecarias alegan como parte de sus políticas de adquisición y valorización mediante una doble justificación: acompañar la demanda de los y las lectoras y las exigencias académicas relativas a las obras femeninas -sobretodo en un contexto post\#MeToo. Estas políticas de adquisición y valorización implican dos formas de clasicización de las escritoras: , mediante la promoción de la literatura contemporánea, y mediante el redescubrimiento de autoras olvidadas, según las prácticas militantes de la segunda ola feminista en Francia.

\section{Entrées d'index}

Mots-clés : auteurs, bibliothèques, littérature contemporaine, bibliothécaires, classicisation, féminisme

Keywords: women writers, libraries, contemporary literature, librarians, classicization, feminism

Palabras clave: autoras, bibliotecas, literatura contemporánea, bibliotecarias, clasicización, feminismo

\section{Notes de la rédaction}

Manuscrit reçu le 7 janvier 2021

Version révisée reçue le 11 juin 2021

Article accepté pour publication le 16 juillet 2021

\section{Texte intégral}

1 En même temps que les femmes acquièrent le droit d'accéder à l'enseignement tout au long du XIXe siècle (Planté, 1989), elles s'emparent de l'écrit non sans bouleverser l'ordre moral et les règles du " champ littéraire » (Bourdieu, 1991). Au cours du XIXe siècle, ce champ se bâtit contre les champs politique et économique pour gagner en autonomie (Bourdieu, 1998) - mais toujours à rebours d'une pratique « féminine » de l'écriture : les écrivaines sont alors disqualifiées, suspectées d'être à l'origine de la décadence d'une littérature pure et désintéressée (Planté, 1989). Force est de constater aujourd'hui la puissance d'un discrédit qui aura évacué du panthéon littéraire nombre de femmes de lettres. Ainsi, les femmes auteures du xixe siècle sont reléguées à des genres mineurs, tels que le journal ou l'ouvrage didactique (ibid.), tandis que l'histoire des écrivaines du xxe siècle semble s'écrire " sur le mode du palimpseste " (Lasserre, 2009 : 43). En même temps que chercheures en littérature et sociologues mettent en lumière la présence des femmes dans le champ littéraire francophone, du Moyen Âge au xxie siècle (Reid, 2020), il a semblé urgent d'analyser, à l'ère contemporaine, les conditions de patrimonialisation (c'est-à-dire de conservation et de transmission) et de classicisation (autrement dit d'élévation au rang de modèle) de la littérature féminine contemporaine. L'étude du travail d'acquisition et de valorisation au sein de la Bibliothèque nationale de France $(\mathrm{BnF})$ permet de penser les conditions de patrimonialisation et de classicisation des écrivaines au sein du champ littéraire en même temps que de mettre au jour celles qui permettent à certaines bibliothécaires de s'engager dans « l'espace de la cause des femmes » (Bereni, 2012). Cette étude apparaît 
nécessaire dans un contexte qui évacue bien souvent, au sein de ce même champ, le travail de passation voire de co-écriture de l'histoire littéraire opéré par les bibliothécaires (Rabot, 2012).

2 La notion de patrimonialisation est alors ici utile en tant qu'outil étatique de conservation et de transmission d'un bien national. La BnF participe de la patrimonialisation des écrivaines, notamment par la voie du dépôt légal qui permet la conservation et la transmission des œuvres. Cependant, si toutes les œuvres publiées en France sont conservées à la $\mathrm{BnF}$ grâce au dépôt légal, seule une partie d'entre elles trouve sa place dans les rayons en libre accès pour le lectorat - une moindre partie encore est mise à l'honneur par la voie de billets de blog, de conférences, etc. Les politiques d'acquisition et de valorisation de la $\mathrm{BnF}$ semblent alors être des marques de " consécration » et des outils de « perpétuation » (Viala, 1993) qui concourent, après la légitimation des prix et des critiques, à la « classicisation » des œuvres.

Pour qu'un ouvrage devienne un classique, il doit être capable de s'ériger en modèle, et, surtout, les institutions littéraires les plus soumises à la volonté politique (bibliothèques, écoles) doivent s'en emparer pour en façonner la réception (ibid.). L'étude des politiques d'acquisition et de valorisation permet donc de penser comment une institution aussi centrale que la $\mathrm{BnF}$ fait entrer les écrivaines dans le patrimoine, mais également comment elle « classicise » certaines œuvres, leur conférant une part de leur grandeur et un statut de modèle.

4 Le rôle des bibliothécaires est en partie déterminé par l'institution au sein de laquelle elles s'inscrivent : celui de la BnF. Cette institution évolue au gré des politiques publiques qui ont permis sa constitution en tant que « bibliothèque de la Nation » en 1790, et son renouvellement, engagé de façon sérieuse à la fin des années 1960 pour la période contemporaine. En effet, dans les années 1960, de premières politiques publiques sont menées, qui confèrent un autre rôle à la Bibliothèque nationale $(\mathrm{BN})$ : celui de la prise en charge d'un public plus large et ainsi d'une formation culturelle hors les murs scolaires et universitaires. C'est alors la naissance d'une « conception moderne d'une bibliothèque entièrement ouverte au public » (Hassenforder, 1993 : 131). Par ailleurs, la question de la démocratisation culturelle, mise à l'agenda politique par Jack Lang dès les années 1980, enjoint les bibliothèques à repenser leur rôle afin de sortir d'une crise financière en inscrivant les productions les moins légitimes dans les propositions de lecture faites au public (comme la bande dessinée, par exemple) (Saez, 1992) ${ }^{1}$. Fort de ces injonctions à l'ouverture au public et à la prise en charge des littératures moins légitimes, François Mitterrand inaugure en 1996 le site Tolbiac, destiné à prendre en charge ce renouvellement des politiques publiques en faveur d'une culture « à la disposition de tous » (Bibliothèque nationale de France). Dans la période plus contemporaine encore, le site Tolbiac s'engage sur plusieurs fronts, et la BnF remplit alors diverses fonctions : elle est le lieu du dépôt légal (loi du 19 mai 1925); un lieu de consultation ouvert à des publics ; elle est également un espace de valorisation et de création événementielle. Enfin, elle est engagée dans la préservation et la valorisation numériques depuis la création du site Gallica en $1997^{2}$.

5 Dans ce contexte, la question de la place des écrivaines au sein des collections de la BnF se pose directement. Tout d'abord en ce que l'institution doit remplir sa fonction encyclopédique, mais également en ce qu'elle se trouve contrainte de répondre à la demande de publics élargis. Il s'agit ainsi pour les agent·e·s engagé.e.s dans les politiques d'acquisition et pour celles et ceux en charge de la valorisation de permettre à la $\mathrm{BnF}$ de rester un haut lieu de conservation encyclopédique où la neutralité des choix est nécessaire pour assurer la représentation de la diversité des productions littéraires et universitaires. En même temps, les bibliothécaires doivent être suffisamment au fait des «tendances » lectorales pour assurer la fonction d'accueil et d'attraction des publics de la BnF.

$6 \quad$ La question de la place de la création littéraire féminine contemporaine est donc un défi pour les agents en charge des acquisitions de monographies et de la valorisation : comment articuler l'injonction sociale à la représentation d'auteures tout en proposant une sélection à prétention " neutre ", qui évacue la question des préoccupations sociales et militantes ? En d'autres termes : comment éthique du service public et sensibilité à la cause des femmes s'articulent-elles au sein des politiques d'acquisition et 
de valorisation des bibliothécaires de la BnF ? Quelles initiatives en découlent et quelles formes prennent ces initiatives?

Cet article se fonde principalement sur des entretiens menés auprès de neuf agentes de la BnF et réalisés entre janvier et juillet 2019. Il a été enrichi par l'exploitation d'une base de données des acquisitions monographiques en littérature française contemporaine. Une base de données recensant l'ensemble des monographies écrites par des écrivaines françaises contemporaines déposées par la voie du dépôt légal entre 1990 et 2018 a également servi à étayer les analyses. Enfin, une étude des billets du blog Gallica et des tweets relatifs aux écrivaines mises à l'honneur en salle ou en ligne vient compléter ce rapport. L'ensemble des données a pu être récolté grâce au concours de la bourse du Comité d'histoire de la BnF.

8 Tout d'abord, il s'agira de questionner l'ethos des bibliothécaires interrogées pour déterminer la contrainte institutionnelle qui pèse sur les choix menés en termes d'acquisition et de valorisation. Par la suite, nous proposerons de penser les possibilités d'agentivité des bibliothécaires interrogées, qui leur permettent de mobiliser ou de réactualiser des ressources qui leur sont propres sans pour autant déroger aux contraintes institutionnelles. Nous verrons notamment dans quels cas et à quelles conditions les bibliothécaires peuvent classiciser la littérature produite par des écrivaines. Nous verrons également quelles contraintes exogènes pèsent sur l'institution tout entière, qui incitent les bibliothécaires à s'engager plus directement dans l'acquisition ou la valorisation des auteures contemporaines. Enfin, nous nous attarderons sur les manières dont les auteures sont intégrées ou réintégrées à la littérature légitime par les bibliothécaires de la $\mathrm{BnF}$, dans un contexte social propice à ces initiatives ${ }^{3}$.

\section{L'ethos bibliothécaire à la BnF : discrétion, neutralité et promotion d'une littérature exigeante}

\section{Discrétion et socialisation des bibliothécaires}

A priori, les formes de «présentation de soi » (Goffman, 1973) des bibliothécaires semblent différer en de nombreux points des autres agents interrogés dans le cadre de précédentes études (Bass, 2019) : les agents littéraires et les éditeurs se distinguent en effet par leur manque de disponibilité, tandis que les bibliothécaires rencontrées ont fait preuve d'un volontarisme singulier. Le temps que les éditeurs et agents littéraires accordent à l'enquêtrice est en effet un temps durant lequel ils ne peuvent pas travailler, tandis que la rencontre avec des chercheurs fait partie des missions des bibliothécaires. Ainsi, la quasi-totalité des personnes sollicitées ont mis entre une journée et une semaine à répondre aux demandes d'entretien, et l'entretien s'est généralement tenu entre une et trois semaines après le premier contact.

10 Pour autant, et si les éditeurs maitrisent leur discours, rendant parfois l'interaction difficile voire impossible, force est de constater l'invariant d'une difficulté à la prise de parole chez les bibliothécaires - la moitié d'entre elles m'a en effet demandé de lui fournir la grille d'entretien avant notre rencontre ; nombreuses sont celles qui ont apporté des notes avec elles ou qui se sont servi de supports externes pour vérifier l'exactitude de leurs propos ; et, enfin, l'une d'entre elles n'a pas souhaité être enregistrée. Le discours des bibliothécaires, à l'inverse de celui des éditeurs, est peu assertif : la prise de parole sur un temps long ne semble pas faire partie de l'habitus bibliothécaire, à plus forte raison lorsqu'on le compare à celui des éditeurs, dont l'identité se construit également sur cette présentation de soi contrôlée, notamment lorsqu'ils ont affaire à l'institution universitaire (ou à l'idée qu'ils s'en font). A contrario, et en dépit d'un volontarisme affiché, les bibliothécaires semblent peu coutumières de l'exercice consistant à parler de soi, ou, si elles le sont, se présentent 
soigneusement et apportent des données scrupuleusement vérifiées afin, peut-être, de ne pas compromettre l'image de l'institution.

Après deux heures d'entretien, l'une des bibliothécaires me confie ainsi avoir « mal à la gorge à force de parler ». Plus directement, une des bibliothécaires explique plusieurs fois n'être « pas à l'aise à l'oral ».

12 Les caractéristiques et les trajectoires sociales des bibliothécaires sont ici à convoquer pour saisir cette moindre assurance dans la prise de parole. Les bibliothécaires interrogées sont toutes des femmes, la quasi-totalité des bibliothécaires en charge des politiques d'acquisition et de valorisation de la $\mathrm{BnF}$ étant des femmes ; à leur poste depuis relativement peu longtemps pour la majorité d'entre elles (entre un an et huit ans, à l'exception près d'une bibliothécaire en poste depuis 26 ans) ; et surtout, leur " griffe » (Bourdieu \& Delsaut, 1975) n'est pas engagée pour la reconnaissance des auteures acquises ou valorisées, l'institution prenant - en partie - le pas sur l'identité singulière de chacune.

Les bibliothécaires ici interrogées ont par ailleurs, pour certaines, bénéficié d'une ascension sociale rare voire inexistante au sein du champ littéraire - dont les fonctions requièrent avant tout la possession d'un capital social important, souvent hérité, et une aisance dans la culture de ce capital (Bass, 2019).

Ces compétences sociales sont probablement moins déterminantes dans la carrière des bibliothécaires, alors majoritairement recrutées parmi la classe moyenne (voire des catégories plus populaires) et dont les trajectoires n'ont pas nécessité d'elles un engagement important dans l'entretien d'un capital social - puisque toutes ont été recrutées par la voie de concours de la fonction publique. En effet, quatre d'entre elles sont issues des classes moyennes ou populaires, au sein desquelles le capital social semble avoir été peu valorisé, à la différence du capital culturel, conçu par la famille, les enseignants et les agentes elles-mêmes comme principal moyen d'ascension sociale. Aussi, les enquêtées sont-elles très diplômées : toutes possèdent au moins un bac +5 , deux d'entre elles sont normaliennes, deux sont docteures et trois sont conservatrices des bibliothèques.

15 Plus spécifiquement, la lecture comme promoteur social a été rapidement valorisée soit par la famille, soit par des enseignants, soit de façon autodidacte. Margaux ${ }^{4}$, encadrante du service Littérature, explique ainsi l'importance que sa mère, pour laquelle « la lecture était un puissant outil de promotion sociale ${ }^{5}$, a accordée aux lectures de ses filles. Cette incitation à la lecture, en dépit d'un milieu social défavorisé, lui a permis de "sauter une classe sociale ». La socialisation littéraire s'est, pour d'autres, faite au travers des enseignants, comme cela a pu être le cas de Léonor ${ }^{6}$, conseillée dans ses lectures par une enseignante et amie de la famille. Ainsi, pour une partie d'entre elles, l'école et la lecture apparaissent comme deux manières privilégiées permettant de gravir l'échelle sociale :

« La lecture étant valorisée sur le plan scolaire, c'est ce qui m’a permis d'être parmi les premières de ma classe. Lorsque l'on aime lire, il est plus facile de réussir dans les disciplines littéraires7. »

Les bibliothèques ont par ailleurs, pour certaines enquêtées, joué un rôle significatif au cours de la socialisation primaire. Elles ont souvent été le premier lieu de rencontre avec la littérature, comme dans le cas de Sylvie ${ }^{8}$ :

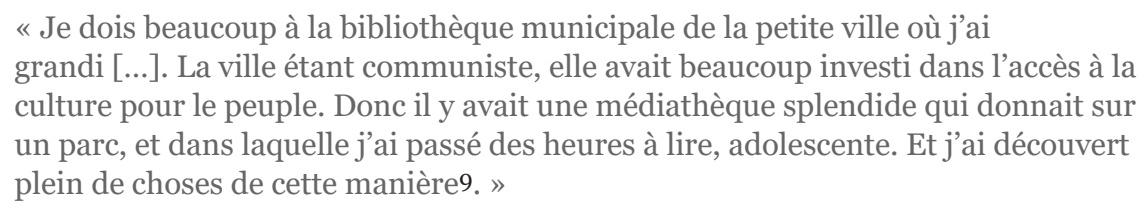
culture pour le peuple. Donc il y avait une médiathèque splendide qui donnait sur un parc, et dans laquelle j'ai passé des heures à lire, adolescente. Et j’ai découvert plein de choses de cette manière9. »

17 La bibliothèque municipale, tout comme l'école, a été un espace de socialisation essentiel pour certaines bibliothécaires, à plus forte raison au sein de milieux sociaux dépourvus de capitaux culturels, économiques ou des deux. Ainsi, Margaux insiste également sur l'importance de la bibliothèque de sa commune, au sein de laquelle les loisirs peu onéreux étaient limités : la bibliothèque à l'abonnement gratuit permettait à ses parents de proposer une activité extrascolaire à leurs filles. Léonor, d'un milieu 
pourtant davantage favorisé, a également d'abord rencontré la littérature grâce à la bibliothèque municipale de sa commune, en région parisienne.

Les bibliothécaires interrogées sont donc, pour la majeure partie d'entre elles, détentrices d'un capital culturel acquis par les voies républicaines (école et bibliothèque municipale) et accordent une importance très relative à la détention d'un important capital social dans la détermination de leur carrière professionnelle. A contrario, elles confèrent un crédit certain aux institutions publiques dans la possibilité de changement social - et ici d'ascension sociale. Elles sont probablement plus à même, dans ces conditions, de défendre l'ethos du service public qu'implique leur fonction. Cet ethos comprend, a priori, l'effacement de certaines dispositions individuelles (comme l'engagement ou le militantisme) à la faveur de la fonction qu'elles endossent, qui les précède et qui perdurera après elles.

\section{La neutralité comme gage d'intégrité intellectuelle}

Ainsi, l'ensemble des bibliothécaires interrogées revendique la neutralité des choix opérés, à plus forte raison dans le cadre des acquisitions monographiques. Les champs lexicaux de la «neutralité », de l'« objectivité », de la primauté d'une démarche « scientifique », et la question de la vertu « encyclopédique » de la BnF traversent l'ensemble des entretiens faits avec des acquéreuses. De cette manière, Léonor confie que « la bibliothèque qui est la [sienne] chez [elle] n'a rien à voir avec ce qu'[elle] achète pour la BnF ». Pour Margaux, l'une des parties essentielles de sa fonction de service public est le travail de "mémoire de la littérature française », c'est-à-dire de conservation et de patrimonialisation par la voie du dépôt légal.

Ne pouvant cependant pas couvrir l'ensemble des champs de production littéraire et scientifique, les acquéreuses réfléchissent en termes de "lacunes » ou de "zones d'ombre », qu'il s'agit de combler pour des raisons d'abord scientifiques, comme l'explique Anne ${ }^{10}$ :

«Lorsqu'on est bibliothécaire, et il s'agit ici d'une forme de continuité du service
public, on est au service d'un domaine : on achète de manière neutre dans un
domaine, avec une vision globale de la collection depuis ses origines. Lorsqu'il y a
des lacunes majeures, notre travail consiste à les combler. En d'autres termes, on
n'achète pas des ouvrages pour faire avancer ses propres recherches ${ }^{11 .}$ »

Ainsi, si le champ académique est davantage axé sur la redécouverte d'auteures, le travail des bibliothécaires sera de rendre compte de cette évolution, mais non de l'impulser, selon Margaux. Elle explique notamment qu'en ce qui concerne la rédaction des notices, ces dernières ne peuvent être révisées que sous l'influence d'études, à l'instar de celles d'Edwige Keller-Rahbé, qui pointe les stratégies de présentation de soi des auteures du xviıe siècle (préférant, souvent temporairement, faire apparaître leur titre ou leur nom d'épouse, ou encore soustraire leur nom à celui du libraire qui les édite, dans la perspective d'être reçues plus favorablement par le lectorat (KellerRahbé, 2010). Selon elle, les chercheurs doivent d'abord s'emparer de telles questions qui doivent devenir " communément admises pour que [leurs] pratiques professionnelles puissent évoluer ».

\section{L'exigence littéraire, stratégie de distinction de la $\mathrm{BnF}$}

22 Enfin, les exigences de l'institution se retrouvent également dans les discours des bibliothécaires : toutes insistent sur la qualité des ouvrages proposés en salle de lecture. Ainsi, Sylvie défend-elle et acquiert-elle principalement de la littérature légitime, en tout cas n'appartenant pas au « sous-champ de grande production » (Bourdieu, 1991 : 7), en se fondant sur le rôle distinctif joué par la BnF dans le paysage des bibliothèques : " il s'agit de permettre aux lecteurs de trouver des auteurs qu'ils ne trouveront peutêtre pas ailleurs », explique-t-elle. 
La BnF semble donc en partie bâtir son identité sur une pratique distinctive en termes d'acquisition et de valorisation : le service public à la BnF implique non seulement la neutralité des choix opérés, l'effacement des particularismes derrière la légitimité des ouvrages préalablement négociée au sein des champs universitaire et littéraire, mais également la promotion d'une littérature que les usagers ne pourraient pas trouver ailleurs et qui justifie alors l'existence même de tels dispositifs d'accueil des publics. Ainsi, la charte documentaire de la BnF mentionne la fonction encyclopédique et l'ambition « universaliste » $(\mathrm{BnF}, 2015: 23)$ des acquisitions, même s'il s'agit d'un « encyclopédisme raisonné » (ibid. : 8) du fait des contraintes budgétaires.

24 Confrontée à l'exigence littéraire, à une discrétion prenant ses racines dans des dispositions lointaines d'autres agents du champ littéraire, et à la neutralité requise par le travail de "facilitatrice ${ }^{12}$ ", la sensibilité à la cause des femmes, à plus forte raison pour une littérature non encore consacrée, est à négocier. Comment les bibliothécaires s'y prennent-elles en discours et en actes ? Quels outils permettent d'ouvrir ces brèches d'agentivité ?

25 En effet, en dépit de discours sur la neutralité, il s'est avéré que la socialisation des bibliothécaires avait beaucoup à voir avec leurs choix d'acquisition et leur engagement dans la valorisation de l'écriture des femmes.

\section{Les espaces d'agentivité dans un système contraint}

\section{Des contraintes endogènes renégociées}

En dépit des contraintes qui pèsent sur la fonction des bibliothécaires, ces dernières semblent disposer d'espaces et d'outils qui leur permettent de négocier habilement entre " exigence de neutralité » requise par le service public et " convictions personnelles » en lien avec les socialisations propres à chacune.

En effet, les acquisitions faites par Sylvie donnent à voir une surreprésentation des femmes (que ce soit dans l'espace «tous publics» ou dans l'espace « recherche ») comparativement au taux d'écrivaines présentes dans le champ littéraire contemporain (d'environ $30 \%$ pour 2006 : Naudier, 2010 : 2). En effet, toutes acquisitions confondues, les écrivaines représentent un total de $36,5 \%$ des acquisitions monographiques (fig. 1 et 2).

Figure 1. Évolution des acquisitions de Sylvie en littérature française contemporaine de 1960 à 2014 en fonction du sexe (en nombre d'ouvrages).

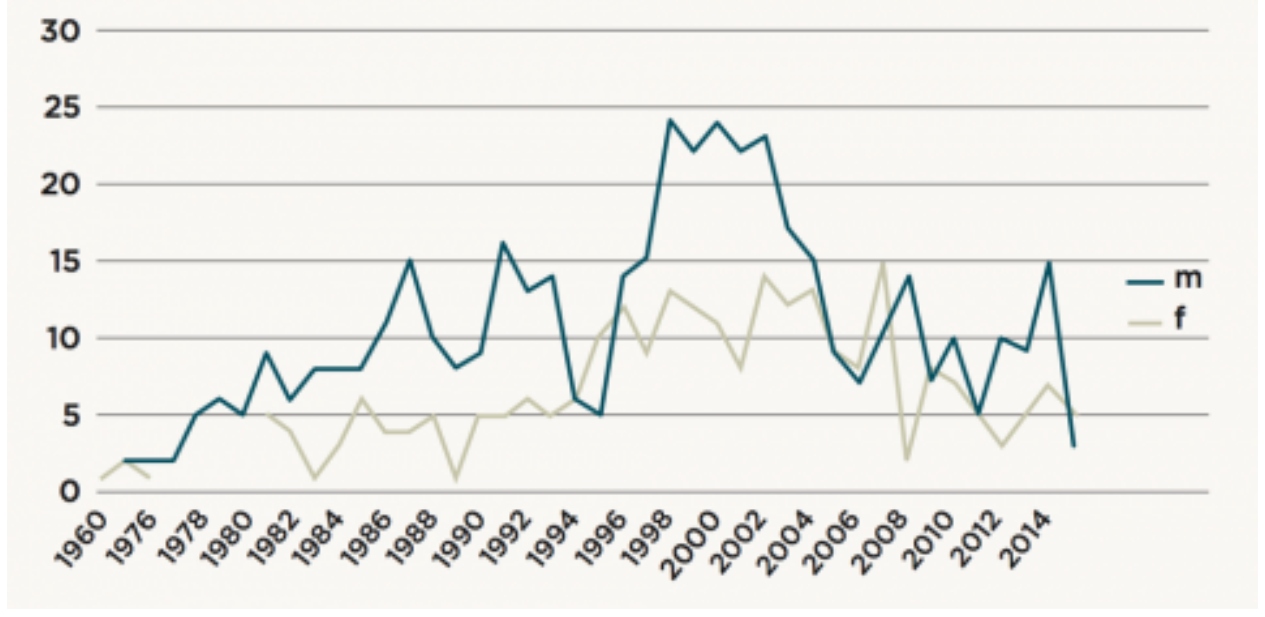

(C) Lilas Bass.

Figure 2. Évolution de la part des acquisitions de littérature contemporaine produite par des femmes de 1960 à 2014 (en \%). 


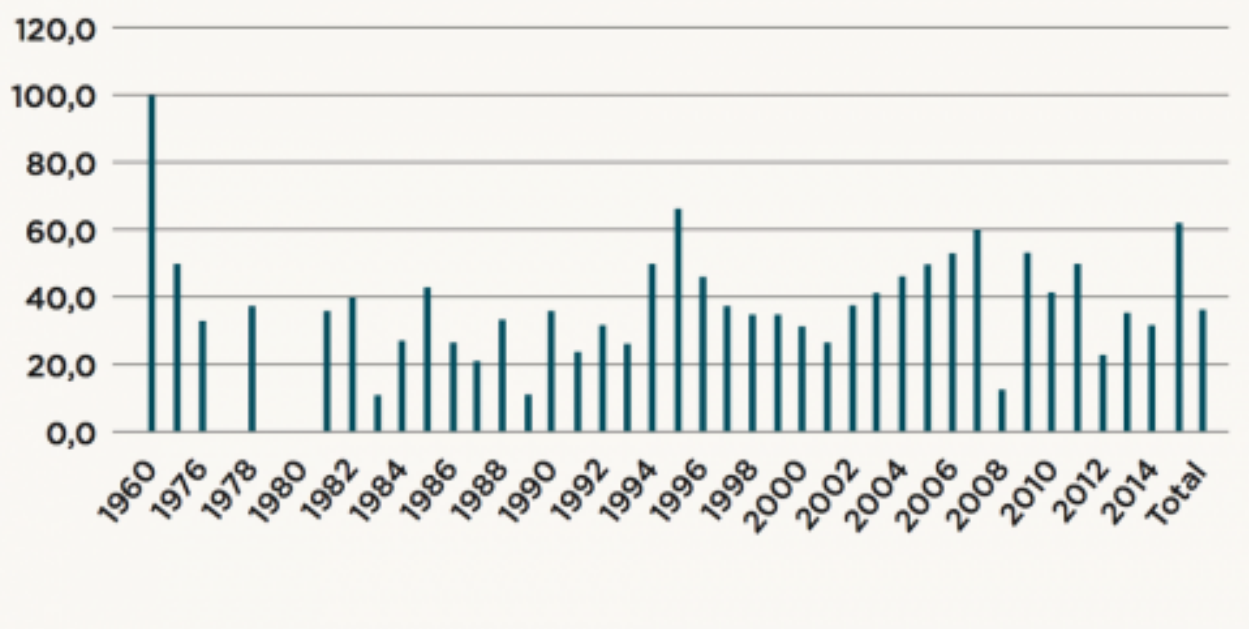

(C) Lilas Bass.

Dans les salles de lecture «tous publics » et « recherche », les femmes sont donc plus que représentées comparativement à leur place dans l'industrie éditoriale. Cela n'est cependant pas sciemment voulu par Sylvie :

« J'en suis ravie, mais j’y ai réfléchi et ce n'est pas conscient. Il y a probablement
un biais, parce qu'en tant que lectrice je vais peut-être aller davantage vers des
livres de femmes, donc je les connais ou je les repère plus facilement ${ }^{13}$. »

Cette dissonance vis-à-vis de la représentation des auteures serait peut-être davantage à mettre en lien avec une socialisation littéraire qui s'est d'abord faite par le biais de lectures autodidactes en bibliothèque municipale ; et d'autre part avec une socialisation féministe par le prisme de la littérature. En témoigne de façon significative sa fréquentation de la librairie des Éditions des Femmes dans les années 1970 :
«À la fin des années 1970, j’ai passé beaucoup de temps, à Lyon, dans la librairie des Éditions des Femmes, qui était vraiment très accueillante. [...] J'allais y passer mes mercredis après-midi. Et je me souviens même que parfois, étant étudiante et ne pouvant pas toujours acheter des livres, on m'avait prêté certains livres. Je les rapportais la semaine d'après. [...] Étant donné que toutes les libraires étaient militantes, elles étaient heureuses de pouvoir faire partager ces lectures à une adolescente. C'est un très bon souvenir, également du point de vue du féminisme14. »

De la même façon, Léonor revendique une sensibilité féministe qui s'est très tôt développée, également par la voie de la lecture d’ouvrages publiés par les Éditions des Femmes. Elle réconcilie ainsi l'exigence de scientificité et les convictions personnelles :

\footnotetext{
« Je pense qu'il n'y a justement pas d'incompatibilité entre l'ethos personnel et professionnel, au contraire. Ce serait problématique si on se mettait effectivement à n'acheter que des textes publiés par des femmes ou sur des femmes. Mais ce n'est pas ce dont il s'agit. C'est qu'il y a quand même assez peu d'études publiées par des chercheurs français de redécouverte d'auteures15. »
}

La nécessaire neutralité et la vocation encyclopédique de leur fonction et de la BnF ne sont ainsi pas remises en cause lorsque les conditions sociales permettent de réactualiser des dispositions acquises antérieurement.

La justification savante qui enjoint à la représentation des auteures ou des intellectuelles oubliées est transversale aux entretiens menés auprès des acquéreuses. Ainsi Anne, également tôt influencée par des lectures féministes (" Ma mère m'avait offert des petits livres publiés par les Éditions des Femmes, dont l’héroïne s'appelait Aurore. [...] Elle faisait ce qu'elle voulait, ces livres permettaient aux jeunes lectrices de se dire : "On peut tout faire" »), justifie-t-elle le travail de redécouverte mené sur certaines figures féminines toujours par la nécessité intellectuelle de la démarche : "C'est une approche scientifique qui m'a guidée, il s'agissait de se demander : "De quelles sources dispose-t-on ? Comment s'appuyer sur les textes ?” » 
La question de la " sensibilité féministe » est pour autant une question transversale à tous les entretiens : toutes les agentes rencontrées dans le cadre de cette étude revendiquent, à divers degrés, une sensibilité voire un militantisme féministe. Cela est sans doute lié au fait que l'ensemble des enquêtées sont des femmes, témoignant d'une féminisation de la profession depuis au moins les années 1950 - $68 \%$ des bibliothécaires étaient en effet des femmes en 2008 (Forlani, 2009). L'« espace de la cause des femmes " (Bereni, 2012) est cependant, dans le cadre des bibliothécaires, contraint par leur fonction et la nécessité de rendre compte de l'état du champ littéraire et du champ universitaire au plus proche de ces deux champs. Cela traverse également tous les entretiens : les bibliothécaires sont des " conservatrices » ou des «facilitatrices », mais en aucun cas des « productrices » de savoir. Pour autant, elles parviennent à négocier entre leur ethos professionnel et certaines socialisations " féministes » par la voie d'une double justification. Tout d'abord en invoquant les travaux universitaires qui encouragent à la redécouverte, ou bien en justifiant un engagement féministe qui serait un engagement strictement intellectuel. Autrement dit, le militantisme féministe au sein de la $\mathrm{BnF}$ ne peut pas prendre une forme traditionnelle de militantisme et ne se dit pas, d'ailleurs, en termes de « militantisme ». Le féminisme trouve sa raison d'être dans des injonctions intellectuelles : oublier les grandes femmes de lettres, c'est manquer à son devoir de transmission des écrits et des savoirs, c'est priver le lectorat d'un riche héritage. Redécouvrir les femmes oubliées (intellectuelles ou femmes de lettres), à l'instar d'un historien, c'est éclairer le passé d'un jour nouveau qui permettra d'enrichir les recherches présentes et futures. Valoriser la création féminine contemporaine, par ailleurs, c'est répondre avant tout à la demande des lecteurs et des lectrices - le \#MeToo est souvent invoqué, tout comme l'est l'ensemble des revendications féministes qui ont émergé depuis. Ainsi, en accompagnant un mouvement social plus général, les bibliothécaires n'outrepassent pas les règles de leurs fonctions, mais les élargissent de façon à concilier " éthique intellectuelle », « service public » et « sensibilité féministe ».

34 Le lieu de la littérature contemporaine s'avère par ailleurs être un espace particulièrement fécond à la réactualisation de dispositions acquises dans d'autres sphères ou à des moments antérieurs de vie. Ainsi, pour justifier la création d'une cote « Xxie siècle » en littérature française, Sylvie écrit dans sa note d’intention :

«Une ouverture sur la littérature contemporaine permet d'offrir au public
universitaire le plaisir, qui n'est pas si fréquent, de la découverte, en lui donnant
l'occasion de trouver au hasard d'un butinage autre chose que ce qu'il est venu
chercher ou que ce qui est directement utile à son travail [...]. Cette possibilité de
surprise est d'autant plus importante que trop d'universitaires, même littéraires,
lisent aujourd'hui assez peu les écrivains contemporains ${ }^{16}$.»

L'ensemble de la note tient parfaitement l'équilibre entre l'accompagnement d'un mouvement déjà engagé à l'université de travail sur la littérature contemporaine et l'incitation active à la découverte d'auteurs encore peu étudiés. Sylvie s'appuie habilement sur son rôle d'accompagnatrice ou de facilitatrice pour l'étendre, dans le cadre de la création de cette nouvelle cote, à un rôle d'incitatrice. Elle rappelle d'ailleurs au cours de l'entretien la solidité universitaire de sa démarche, notamment pour penser les bornes d'une cote de littérature contemporaine - qui débute en 1980, suivant les recommandations de Dominique Viart et Bruno Vercier (2005).

36 En ce qui concerne les liens entretenus avec les industries éditoriales, ils semblent a priori du même ordre : Sylvie suit ainsi le calendrier éditorial, indiquant qu'une grande partie des acquisitions se fait au moment de la rentrée littéraire. En même temps, elle se positionne (et le cadre d'une cote " vivante » permet d'autant mieux l'extension de ses prérogatives) contre certains choix éditoriaux.

37 Encouragées par des politiques publiques qui enjoignent la prise en charge de cette littérature à la $\mathrm{BnF}$, les bibliothécaires disposent alors d'un endroit où le « classique » n'est pas entièrement établi, et au sein duquel elles participent largement à sa définition. Plus que simples "facilitatrices ", les bibliothécaires engagées dans l'acquisition et la valorisation d'une littérature contemporaine disposent de marges de 
manœuvre importantes pour convoquer des ressources acquises en dehors de leurs fonctions.

La BnF semble ainsi faire partie, dans une certaine mesure, des " appareils de classicisation » (Viala, 1993 : 23) : si l'école ou les universités ont sélectionné la majorité des classiques, la BnF peut, par exemple, proposer des bibliographies sélectives composées par ses agent·e.s, notamment lors des concours d'agrégation. Cette sélection bibliographique encadre la réception d'une œuvre et érige certains travaux en classiques, comme c'est le cas, par exemple, de la bibliographie accompagnant l'œuvre de Marguerite de Navarre, au programme de l'agrégation de lettres modernes en 2021, et comprenant 24 pages de références bibliographiques qui viennent compléter les indications données par le ministère de l'Éducation nationale (BnF, 2021a).

Pour le domaine des lettres contemporaines, la $\mathrm{BnF}$ organise des master classes depuis 2016, consacrant ainsi chaque année un·e auteur.rice français·e et participant d'une forme de classicisation en ce qu'elle érige les intervenant.e-s des master classes en modèles pour quiconque voudrait écrire :

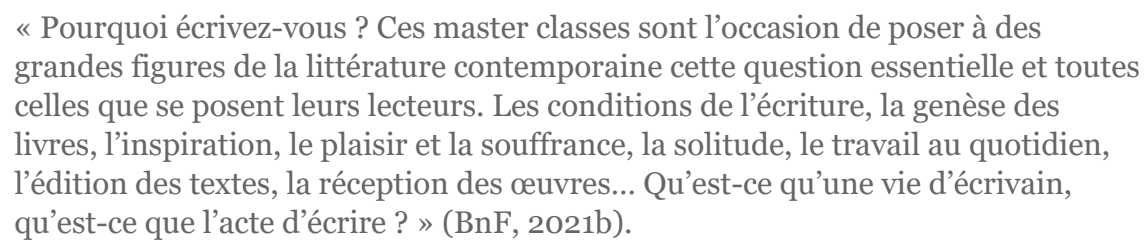
encore l'écrivaine et illustratrice de BD Pénélope Bagieu.

\section{Des contraintes exogènes : demande du public et nécessité intellectuelle en contexte de quatrième vague féministe}

La possibilité de réactualiser goûts et compétences acquises en dépit d'une éthique de la neutralité scientifique n'est cependant pas le seul fait des bibliothécaires : en effet, la période étudiée donne à voir le renforcement d'un tournant engagé par la BnF dans la prise en compte des personnes fréquentant les salles " tous publics », qui permettent aux agent·e.s engagéee-s dans les acquisitions et la valorisation de négocier plus facilement la place de la littérature contemporaine.

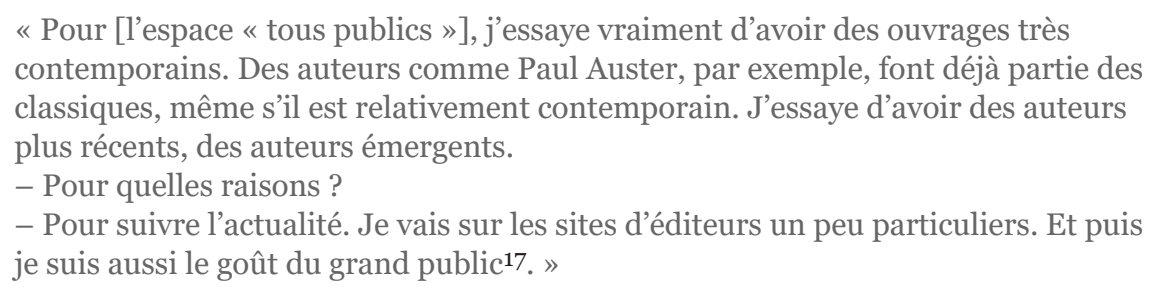

La « crise sociale » post-\#MeToo, à savoir l'engouement pour la cause des femmes qui a émergé à la suite du mouvement en ligne \#MeToo en 2017, semble également avoir contraint les divers champs à prendre position et à répondre à l'urgence sociale de l'égalité femmes-hommes : au sein des bibliothèques, cette demande était prise en compte avant la réactivation et les prémices d'une institutionnalisation des revendications féministes, comme en témoigne notamment l'engagement de Sylvie en faveur de la création féminine. Cependant, le post-\#MeToo semble avoir accéléré l'injonction à la représentation d'auteures. Aussi, Margaux remarque-t-elle un accroissement significatif de la consultation de certaines publications en études féministes depuis 2017, qu'elle tente de systématiser pour pouvoir mieux répondre à la demande sociale : « J'ai vu qu'il y avait seize lecteurs de Planté (Planté, 1989) ${ }^{18}$ en 2017. 
Il serait intéressant qu'on en ait en salle parce que ça permet à deux lecteurs d'y avoir accès ", explique-t-elle.

Cependant, cet engagement de la part des agent·e·s de la $\mathrm{BnF}$ a été plus largement publicisé (et plébiscité) à partir de 2018, notamment par la voie du numérique. Ainsi, si Sylvie, alors collaboratrice dans l'animation du compte Twitter de la BnF, a publié des tweets relatifs aux auteures avant le \#MeToo et l'engouement féministe qui s'en est suivi, ces tweets ont reçu un accueil bien plus favorable à partir de 2018. À titre d'exemple, elle fait valoir dès 2016 les auteures mises à disposition par la BnF dans le cadre du Hackathon ${ }^{19}$, et le tweet est " liké » 31 fois et « retweeté » douze fois ; tandis que le tweet d'avril 2018 proposant un lien vers la biographie de Marie-Louise Gagneur ${ }^{20}$ est « liké » 127 fois et « retweeté » 94 fois dans le cadre du débat sur la féminisation des titres et métiers vis-à-vis duquel l'Académie française était (à nouveau) chargée de prendre position au printemps 2018.

De la même façon, le numérique, à plus forte raison dans un contexte de quatrième vague féministe d'abord impulsée par la voie des réseaux sociaux, est investi par Nathalie dans ses projets de valorisation, notamment via le projet d'encyclopédie en ligne Digitens, au sein duquel elle souhaite "mettre à l'honneur les femmes de lettres anglaises » en coopération avec le National Archives de Londres ${ }^{21}$.

\section{Les conséquences sur la représentation des femmes dans la littérature}

\section{Les femmes surreprésentées dans les genres consacrés en littérature contemporaine}

Les diverses exigences institutionnelles et les brèches au sein desquelles les bibliothécaires peuvent renégocier la définition des classiques donnent lieu à des politiques d'acquisition et de valorisation qui vont dans le sens du progrès social sans remettre en cause l'exigence de qualité des œuvres proposées par la BnF au public : aussi, les écrivaines acquises et promues le sont-elles dans des genres littéraires très légitimes dans le champ littéraire. En littérature française contemporaine, les poétesses sont ainsi mises à l'honneur, et des corpus de poétesses sont composés, souvent accompagnés de l'appareil critique afférent. En effet, si l'on s'attarde sur les acquisitions monographiques faites par Sylvie, il est curieux de noter l'importance accordée à la poésie et à la relative importante place des femmes dans ce genre littéraire, pourtant connu pour être historiquement excluant parce que très codifié (Sapiro, 2011 : 181).

Figure 3. Part des écrivaines en libre accès (espace " tous publics ») en littérature contemporaine en fonction du genre littéraire.

\begin{tabular}{|l|l|l|l|l|}
\hline Genre littéraire/Sexe & F & H & Total & Ratio \\
\hline Roman & 257 & 387 & 644 & $40 \%$ \\
\hline Poésie & 37 & 90 & 127 & $29 \%$ \\
\hline Théâtre & 7 & 15 & 22 & $32 \%$ \\
\hline Bibliographies & 1 & 0 & 1 & $100 \%$ \\
\hline Autres genres & 0 & 10 & 10 & $0 \%$ \\
\hline Genre non spécifié & 66 & 123 & 189 & $35 \%$ \\
\hline
\end{tabular}


Il semblerait donc que la promotion d'une littérature légitimée par son genre n'entre pas en contradiction avec l'identité de la BnF. Aussi, Sylvie acquiert-elle des poétesses nées après les années 1970, telles Emmanuelle Le Cam, Virginie Poitrasson, Laurine Rousselet, Gwenaëlle Stubbe, Sereine Berlottier ou Virginie Lalucq; ou bien met-elle à l'honneur les poétesses contemporaines dans la salle « tous publics » à l'occasion de la 20e édition du Printemps des poètes en mars $2018^{22}$.

$47 \quad$ Si l'on compare les genres littéraires investis par les écrivaines et ceux représentés par la BnF, le décrochage est flagrant : ainsi, sur le total des titres écrits en France par des femmes et publiés en France entre 1990 et 2018, 12 \% appartiennent à la " romance » et $9 \%$ à l'édition numérique ${ }^{23}$. L'un des principaux facteurs qui peut expliquer cette surreprésentation des auteures dans ces genres est celui de marchés particulièrement dynamiques dans ces secteurs, et notamment celui de la jeunesse, quatrième secteur en termes de part du marché du livre (SNE, $2018: 11$ ). Cela conduit à penser que la BnF n'a pas vocation à classiciser des genres jugés peu légitimes dans le champ littéraire. Dans le cadre de la littérature produite par des écrivaines, à plus forte raison peut-être, la " classicisation » ne semble pouvoir se négocier qu'au sein de genres légitimes afin de permettre à la BnF de toujours se positionner comme force de proposition d'une littérature exigeante.

\section{Une redécouverte des auteures oubliées}

Une autre voie permet également de répondre aux attentes du lectorat en même temps qu'à l'exigence de qualité des œuvres proposées : celle de la "redécouverte » d'écrivaines oubliées mais que le temps a, au moins en partie, classicisées. Cette politique de redécouverte est en tous points peu risquée pour la $\mathrm{BnF}$ en même temps que demandée par le public : la remise en question des œuvres « ayant fait œuvre » est moins difficile à négocier dans le cadre d'une littérature déjà sélectionnée par l'histoire littéraire. Le cycle « femmes de lettres » du blog Gallica de la BnF est alors un outil qui permet de réconcilier " prérogatives institutionnelles ", " attentes sociales » et « sensibilité féministe ».

En même temps, les routines de la redécouverte historique sont, depuis les années 1970, incorporées dans l'habitus intellectuel (au moins féministe) : il semble en effet ici que se rejouent des enjeux et des manières de faire propres à la deuxième vague féministe qui, si elle a souffert de son manque d'institutionnalisation, a mis en place des pratiques intellectuellement justifiables pour valoriser la place des femmes dans l'histoire. Ainsi, Anne cite-t-elle spontanément les approches proposées par Michelle Perrot (Perrot \& Duby, 1991-1992) pour justifier sa propre démarche, et Léonor de remarquer : " J'ai le sentiment qu'en ce moment, il y a un mouvement de relecture de l'histoire littéraire. »

\section{Conclusion}

En dépit d'un travail invisibilisé dans le champ littéraire et d'une fonction exercée sous contraintes, les bibliothécaires de la BnF négocient habilement la détention d'un véritable pouvoir quant à la classicisation de la littérature produite par des écrivaines. Ainsi, si les bibliothécaires se soumettent à certaines conditions de classicisation telles que la reconnaissance préalable par d'autres instances de consécration (maisons d'édition, critiques littéraires et travaux universitaires), il n'en demeure pas moins qu'elles jouent un rôle dans la co-écriture de l'histoire littéraire. Leur fonction d'accompagnement des publics leur enjoint de créer les conditions de réception des œuvres féminines, dont les plus légitimes (souvent issues de genres légitimes) sont mises à l'honneur en ligne, par exemple par la rédaction d'un billet biographique. En ce qu'elles déterminent en partie l'« horizon d'attente » (Jauss, 1990), et donc la réception d'une œuvre, les bibliothécaires participent de sa classicisation. 
Deux voies principales sont alors empruntées par les bibliothécaires de la BnF qui concourent à la classicisation des œuvres féminines : d'une part, outre un effort de patrimonialisation mené par l'ensemble de l'institution par la voie du dépôt légal, les bibliothécaires acquièrent et valorisent une littérature contemporaine au sein de laquelle le classique est encore à construire ; d'autre part, elles réactualisent les procédés féministes de révision de l'histoire littéraire, à la faveur des femmes de lettres oubliées.

\section{Bibliographie}

Bass (Lilas). 2019. "L'éditeur de littérature consacrée face au chercheur en sciences sociales : dits, non-dits et contrepoints heuristiques ». Mémoires du livre / Studies in Book Culture [en ligne], 10(2), "Les discours de l'éditeur / The Publisher's Discourse », sous la direction d'Anthony Glinoer, p. 1-27 : https://www.erudit.org/fr/revues/memoires/2019-v10-n2memoires04677/1060970ar/ [consulté le 9 septembre 2021].

Bereni (Laure). 2012. « Penser la transversalité des mobilisations féministes : l'espace de la cause des femmes ", p. 27-41 in Les Féministes de la deuxième vague / sous la direction de Christine Bard. Rennes : PUR (Archives du féminisme).

BnF [Bibliothèque nationale de France]. 2015. « Charte documentaire. Politique d'enrichissement des collections ", site de la BnF : https://www.bnf.fr/sites/default/files/201811/Charte\%2odocumentaire\%202015.\%20Version\%2ointégrale.pdf [consulté le 9 septembre 2021].

BnF [Bibliothèque nationale de France]. 2021a. "Bibliographies pour l'agrégation 2021. Agrégation de lettres modernes : Marguerite de Navarre », site de la BnF, rubrique « Préparer les agrégations de lettres $»:$ https://www.bnf.fr/sites/default/files/202011/biblio\%20agreg\%202021\%20marguerite\%2ode\%20navarre.pdf [consulté le 9 septembre 2021].

BnF [Bibliothèque nationale de France]. 2021b. « Master classes littéraires », site de la Bnf, rubrique " Découvrir / Départements de collections / Littérature et art 》 : https://www.bnf.fr/fr/masterclasses-litteraires [consulté le 9 septembre 2021].

Bourdieu (Pierre). 1991. "Le champ littéraire ». Actes de la recherche en sciences sociales, 89, p. $3-46$.

DOI : 10.3917/arss.p1991.89n1.0003

Bourdieu (Pierre). 1998. Les Règles de l'art. Genèse et structure du champ littéraire. Paris : Seuil.

Bourdieu (Pierre) \& Delsaut (Yvette). 1975. « Le couturier et sa griffe : contribution à une théorie de la magie ». Actes de la recherche en sciences sociales, 1(1), p. 7-36.

DOI : $10.3406 /$ arss.1975.2447

Forlani (Séverine). 2009. Femmes, pouvoir et bibliothèques : L'accès aux hautes fonctions dans les bibliothèques françaises. Mémoire pour le diplôme de conservateur de bibliothèque. En ligne : https://www.enssib.fr/bibliotheque-numerique/documents/48195-femmes-pouvoir-etbibliotheques.pdf [consulté le 9 septembre 2021].

Goffman (Erving). 1973. La Mise en scène de la vie quotidienne. Vol. 1 : La Présentation de soi. Traduit de l'anglais par Alain Accardo. Paris : Minuit (Le sens commun).

Hassenforder (Jean). 1993. "Notes critiques : Martine Poulain (dir.). 1992. Histoire des bibliothèques françaises. T. IV : Les Bibliothèques au xxe siècle (1914-199o) ». Revue française de pédagogie, 103, p. 130-132.

Jauss (Hans Robert). 1990. Pour une esthétique de la réception [1978]. Traduit de l'allemand par Claude Maillard. Paris : Gallimard (Tel).

Keller-Rahbé (Edwige). 2010. «Pratiques et usages du privilège d'auteur chez Mme de Villedieu et quelques autres femmes de lettres au Xvire siècle ». CEuvres \& Critiques, 35(1), p. 69-94.

Lasserre (Audrey). 2009. « Les femmes du xxe siècle ont-elles une histoire littéraire ? ». Les Cahiers du Cerrac, 4, p. 38-54.

Naudier (Delphine). 2010. « Les écrivaines et leurs arrangements avec les assignations sexuées ». Sociétés contemporaines, 78(2), p. 39-63.

DOI : $10.3917 /$ soco.078.0039

Perrot (Michelle) \& Duby (Georges). 1991-1992. Histoire des femmes en Occident, 5 vol. Paris : Plon.

Planté (Christine). 1989. La Petite Sœur de Balzac. Essai sur la femme auteur. Lyon : PUL.

DOI : $10.4000 /$ books.pul.22527

Rabot (Cécile). 2012. « Bibliothécaires en quête de reconnaissance ». Savoir/Agir, 20, p. 91-96. 
Reid (Martine). 2020. Femmes et littérature. Une histoire culturelle, 2 vol. Paris : Gallimard (Folio essais).

DOI : 10.3917/gall.reid.2020.02

Saez (Guy). 1992. " Politiques culturelles, lecture publique et décentralisation », p. 366-379 in Histoire des bibliothèques françaises. T. IV : Les Bibliothèques au Xxe siècle (1914-1990) / sous la direction de Martine Poulain. Paris : Promodis / Éditions du Cercle de la Librairie.

Sapiro (Gisèle). 2011. La Responsabilité de l'écrivain. Littérature, droit et morale en France (XIXe-XXIe siècle). Paris : Seuil.

DOI : $10.14375 / \mathrm{NP} .9782021032888$

SNE [Syndicat national de l'édition]. 2018. Les Chiffres de l'édition 2017-2018. Rapport statistique $d u$ SNE. Paris : SNE. En ligne : https://www.sne.fr/app/uploads/2018/o7/RS18_BatWEBSignet.pdf [consulté le 9 septembre 2021].

Viala (Alain). 1993. «Qu'est-ce qu’un classique ? ». Littératures classiques, 19, p. 11-31.

DOI : 10.3406/licla.1993.1737

Viart (Dominique) \& Vercier (Bruno). 2005. La Littérature française au présent. Héritage, modernité, mutations. Paris : Bordas.

\section{Notes}

1 Notamment au travers du plan intitulé « L’impératif culturel » et porté par Jack Lang dès 1981 (Saez, 1992 : 479).

2 Voir « Histoire de la Bibliothèque nationale de France », site de la BnF, rubrique « Découvrir / Histoire et missions »: https://www.bnf.fr/fr/histoire-de-la-bibliotheque-nationale-de-france (consulté le 9 septembre 2021).

3 Cet article se fonde principalement sur des entretiens menés auprès de neuf agentes de la BnF et réalisés entre janvier et juillet 2019. Il a été enrichi par l'exploitation d'une base de données des acquisitions monographiques en littérature française contemporaine. Une base de données recensant l'ensemble des monographies écrites par des écrivaines françaises contemporaines déposées par la voie du dépôt légal entre 1990 et 2018 a également servi à étayer les analyses. La première base de données, "Acquisitions d'œuvres par le département de littérature contemporaine de 1960 à 2014 ", a été construite à partir d'un fichier Excel recensant les œuvres achetées par la chargée des acquisitions de ce même département. Une synthèse de ces acquisitions figure ici sous forme de tableau (fig. 1). La deuxième base de données, "Euvres écrites par des femmes et déposées au département littérature et arts de la BnF entre 1990 et 2018 », comprend toutes les œuvres féminines recensées par la BnF par la voie du dépôt légal entre les dates mentionnées. Cette base a été constituée à partir d'un travail d'extraction des cotes réalisé par une agente du département des métadonnées. Elle permet de connaître précisément la production littéraire féminine à partir des années 1990. Enfin, une étude des billets du blog Gallica et des tweets relatifs aux écrivaines mises à l'honneur en salle ou en ligne vient compléter ce rapport. L'ensemble des données a pu être récolté grâce au concours de la bourse du Comité d'histoire de la BnF. Les prénoms de l'ensemble des enquêtées ont été modifiés.

4 Encadrante du service Littérature, en poste depuis 2 ans et demi, F, 38 ans, bac +5 (ENS), conservatrice.

5 Entretien réalisé le 23 mars 2019 à la BnF.

6 Chargée d'acquisition littérature générale et littérature comparée, écrivaine, en poste depuis 5 ans, F, 48 ans, bac +5 en lettres modernes, conservatrice.

7 Entretien réalisé avec Margaux le 21 mars 2019 à la BnF.

8 Chargée des acquisitions en littérature française contemporaine, du dépôt légal du web et de la valorisation numérique du département littérature et art, en poste depuis 26 ans, F, 56 ans, bac +8 en littérature (ENS de Saint-Cloud), agrégée de lettres.

9 Entretien réalisé le 25 janvier 2019 à la $\mathrm{BnF}$.

10 Chargée de collection en lettres classiques, latin et grec ancien, coordination et valorisation, en poste depuis 3 ans, F, 40 ans, bac +8 en égyptologie, bibliothécaire.

11 Entretien réalisé le 21 mars 2019 à la BnF.

12 Pour reprendre le terme utilisé par Anne lors de notre entretien.

13 Extrait d'entretien avec Sylvie.

14 Extrait d'entretien avec Sylvie.

15 Extrait d'entretien avec Léonor.

16 Extrait d'une note d'intention rédigée par Sylvie : "Création d'une cote " xxie siècle " ", 13 mai 2001.

17 Extrait d'entretien avec Nathalie. 
18 Ouvrage sur l'invisibilisation et le discrédit de la création littéraire féminine au xIXe siècle. Margaux indique ici qu'après l'étude des prélèvements en magasin pour l'année 2017, La Petite Sœur de Balzac de Planté a été consulté par seize lecteur·rice-s. à ce titre, il lui parait légitime que l'ouvrage soit également proposé dans l'espace « tous publics ».

19 Voir : https://twitter.com/laBnF/status/799975538421010433 (consulté le 9 septembre 2021).

20 Voir : https://twitter.com/laBnF/status/1011625307059769344 (consulté le 9 septembre 2021).

21 Un autre aspect de la recherche serait probablement à questionner plus avant : celui du rôle joué par le lectorat, dont les préférences semblent participer, au moins depuis les années 1990 et l'ouverture d'un site ouvert au grand public, au processus de classicisation.

22 Voir : https://twitter.com/laBnF/status/969974823295373312 (consulté le 9 septembre 2021).

23 Ces statistiques ont été produites à partir des métadonnées du dépôt légal des ouvrages produits en France par des écrivaines entre 1990 et 2018. Les métadonnées permettant d'identifier, entre autres, la maison de publication, j'ai regroupé les ouvrages en fonction des types de maison dans lesquels ils étaient publiés pour approcher les genres littéraires dans lesquels les écrivaines se retrouvaient le plus fréquemment (par exemple, pour la jeunesse, j’ai réuni, entre autres, les titres parus chez Harlequin). La densité de la base de données m'a cependant contrainte à ne traiter que les maisons d'édition ayant publié au moins 500 titres entre 1990 et 2018, ce qui invisibilise les petites structures.

\section{Table des illustrations}

\begin{tabular}{|c|c|c|}
\hline \multirow{2}{*}{\multicolumn{2}{|c|}{$\begin{array}{l}\text { Titre } \\
\text { Crédits }\end{array}$}} & $\begin{array}{l}\text { Figure 1. Évolution des acquisitions de Sylvie en littérature française } \\
\text { contemporaine de } 1960 \text { à } 2014 \text { en fonction du sexe (en nombre } \\
\text { d'ouvrages). }\end{array}$ \\
\hline & & (C) Lilas Bass. \\
\hline \multirow{6}{*}{ 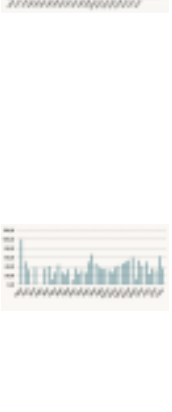 } & ئيL & $\begin{array}{l}\text { http://journals.openedition.org/culturemusees/docannexe/image/6802/img- } \\
\text { 1.png }\end{array}$ \\
\hline & Fichier & image/png, 40k \\
\hline & Titre & $\begin{array}{l}\text { Figure 2. Évolution de la part des acquisitions de littérature } \\
\text { contemporaine produite par des femmes de } 1960 \text { à } 2014 \text { (en \%). }\end{array}$ \\
\hline & Crédits & (C) Lilas Bass. \\
\hline & UيR & $\begin{array}{l}\text { http://journals.openedition.org/culturemusees/docannexe/image/6802/img- } \\
\text { 2.png }\end{array}$ \\
\hline & Fichier & image/png, 34k \\
\hline
\end{tabular}

\section{Pour citer cet article}

Référence papier

Lilas Bass, « La classicisation des écrivaines à la Bibliothèque nationale de France : étude des politiques d'acquisition et de valorisation des œuvres produites par des femmes », Culture \& Musées, 38 | 2021, 99-122.

Référence électronique

Lilas Bass, « La classicisation des écrivaines à la Bibliothèque nationale de France : étude des politiques d'acquisition et de valorisation des œuvres produites par des femmes », Culture \& Musées [En ligne], 38 | 2021, mis en ligne le 10 novembre 2021, consulté le 08 décembre 2021. URL : http://journals.openedition.org/culturemusees/6802 ; DOI :

https://doi.org/10.4000/culturemusees.6802

\section{Auteur}

\section{Lilas Bass}

Lilas Bass, EHESS (CESSP), Paris 1 Panthéon-Sorbonne

Diplômée d'un master en politiques culturelles de Sciences Po Grenoble et d'un master « Genre, politique et sexualité » de l'École des hautes études en sciences sociales (EHESS), Lilas Bass est doctorante en sociologie à l'EHESS sous la direction de Gisèle Sapiro. Son doctorat porte sur le rôle des intermédiaires littéraires dans la publication, la publicisation et la valorisation de la littérature produite par les écrivaines contemporaines de langue française (de 1980 à nos jours). ATER à Paris 1, elle participe par ailleurs à une enquête collective sur les agents littéraires 
internationaux, coordonnée par Gisèle Sapiro (CNRS-EHESS) et Tristan Leperlier (CNRS-

EHESS). Elle a bénéficié d'une bourse du Comité d'histoire de la BnF en 2018-2019, qui lui a permis de mettre au jour certaines conditions de patrimonialisation des écrivaines au sein de cette institution. Elle est l'auteure de : "La "putain" des éditions du Seuil : publication,

publicisation et réception(s) des œuvres de Nelly Arcan dans le champ littéraire français » (dans Prostitution, travail du sexe et écriture, sous la direction d'Isabelle Boisclair, à paraître en 2021) ; " Les Éditions Verticales. Sociohistoire de la construction d'une position emblématique dans le champ littéraire (1997-2017) » (dans Écritures contemporaines, la revue des lettres modernes, à paraître en 2021). Elle a également publié : « L'éditeur de littérature consacrée face au chercheur en sciences sociales : dits, non-dits et contrepoints heuristiques ».

Courriel : lilas.bass[at]gmail.com

\section{Droits d'auteur}

Culture \& Musées 